\title{
NUEVO ATLAS LINGÜÍSTICO ETLEN, SOBRE LA FRONTERA ENTRE EL GALLEGOPORTUGUÉS Y EL ASTURLEONÉS EN ASTURIAS
}

\author{
Ramón de Andrés Díaz \\ Universidad de Oviedo (Seminariu de Filoloxía Asturiana) \\ http://dx.doi.org/10.18778/8220-201-4.05
}

\section{Resumen}

El ETLEN es un nuevo atlas centrado en una zona de frontera lingüística, la que separa los dominios del gallegoportugués y asturleonés en el occidente de Asturias. La fuente de datos es una encuestación propia en 40 puntos del territorio. Además de una sección dialectográfica o de cartografía dialectal clásica (531 mapas correspondientes a otras tantas isoglosas), incluye dos novedosas secciones de cartografía matemático-estadística: horiométrica o de adscripción geotipológica de geovariantes (35 mapas); y dialectométrica o de medición de semejanzas entre los puntos del territorio (77 mapas).

Palabras clave: Atlas lingüístico, gallegoportugués, asturleonés, horiometría, dialectometría.

En la parte más occidental de Asturias, limítrofe con Galicia, hay una conocida frontera entre dos dominios lingüísticos, el del gallegoportugués y el del asturleonés. Se trata de la zona del Eo-Navia, llamada así por los dos ríos que la delimitan. En ella encontramos un conjunto de hablas conocidas genéricamente como gallego-asturiano, gallego de Asturias, eonaviego o "fala”, entre otras denominaciones. Esa frontera geolectal está formada por un haz de isoglosas muy conocido en la dialectología hispánica y románica. 
En el año 2000 comenzó el proyecto Estudio de la Transición Lingüística en la Zona Eo-Navia (ETLEN), emprendido por un equipo de investigadores de la Universidad de Oviedo. Su objetivo era conocer en profundidad cómo se estructura dicha frontera geolectal y qué características presenta. Se hizo un estudio de campo pormenorizado, investigando centenares de isoglosas geográficas y practicando métodos de tipo estadístico. Fruto de ello es la publicación, a finales del año 2017, del atlas lingüístico Estudiu de la Transición Llingüística na Zona Eo-Navia (ETLEN). Atles llingüisticu dialectográficu - horiométricu - dialectométricu (Andrés Díaz et al., 2017).

\section{1.}

\section{Frontera y adscripción geolingüística}

En la Península Ibérica existe un conjunto de dominios lingüisticos. Enumerados de oriente a occidente son: el catalán, el occitano (aranés), el aragonés, el vasco, el castellano, el asturleonés y el gallegoportugués. Entre ellos existen límites o fronteras, que pueden ser más o menos abruptas o graduales. En la franja más occidental de Asturias encontramos el tramo más septentrional de la frontera entre el gallegoportugués y el asturleonés. Entre lenguas semejantes o emparentadas, la frontera suele ser gradual (progresiva, continua, difusa), formando zonas o áreas de transición, que se manifiestan en forma de contínuums geolectales. La frontera del Eo-Navia es de este tipo. Por tanto, el Eo-Navia es una zona de transición lingüistica, donde se habla un gallegoportugués de transición al asturleonés. Los primeros estudiosos que incluyeron estas hablas dentro del gallegoportugués fueron Munthe (1887) y Menéndez Pidal (1906), y desde entonces, y con el avance de los conocimientos dialectológicos de la zona, esa adscripción geotipológica ha venido siendo prácticamente unánime entre los estudiosos; sobre este asunto se puede encontrar amplia información en Andrés Díaz et al. (2017: 9-13). 


\section{Algunos conceptos dialectológicos}

Seguidamente enumeramos una serie de conceptos importantes en la metodología del proyecto y atlas ETLEN (Andrés, 2011, 2015; Andrés Díaz et al., 2013; Andrés Díaz et al., 2017: 13-15):

(1) Fenómeno diferencial: cualquier fenómeno lingüístico susceptible de presentar variación dialectal. Por ejemplo, "conservación o pérdida de la -n-intervocálica del latín".

(2) Rasgo diferencial o geovariante: cada una de las variaciones dialectales de ese fenómeno. El mencionado fenómeno tiene dos geovariantes en nuestra zona:

- La geovariante (a) con distribución occidental: pérdida de -n-, dando lugar a la forma ter.

- La geovariante (b) con distribución oriental: mantenimiento de -n-, de donde resulta la forma tener.

(3) Campo de variación es el conjunto de las geovariantes adscritas al mismo fenómeno diferencial.

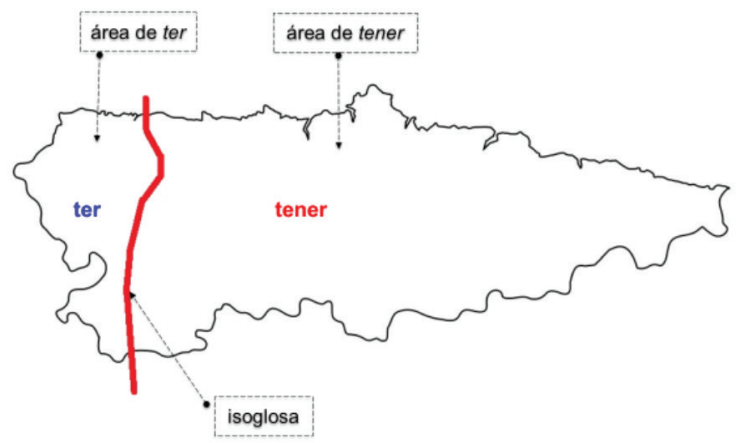

Figura 1. Fenómeno lingüístico diferencial "conservación o pérdida de -nintervocálica latina”.

(4) Área. Territorio geográfico que corresponde a cada geovariante.

(5) Isoglosa. Línea imaginaria que separa dos áreas adscritas al mismo fenómeno diferencial. 
(6) Haz de isoglosas. Acumulación de isoglosas que siguen recorridos muy cercanos a lo largo de la misma zona geográfica. Y así, en el Eo-Navia la isoglosa -n- intervocálica está acompañada de otras muchas que discurren más o menos cercanas a ella.

(7) Frontera lingüística. La existencia de un haz de isoglosas en una zona determinada es la manifestación de una frontera lingüística gradual. Esto significa que en esa zona se acumulan muchas diferencias lingüísticas por unidad de distancia, lo cual indica el paso gradual, pero rápido, de un dominio lingüístico a otro.

(8) Dominios lingüísticos. Son los espacios geográficos separados a ambos lados por la frontera, es decir, por el haz de isoglosas.

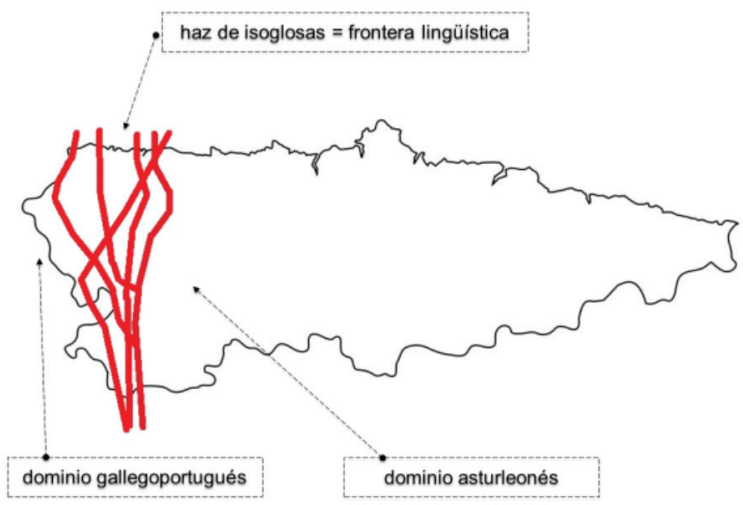

Figura 2. Haz de isoglosas y dominios lingüísticos que separa.

\section{3.}

\section{Características del atlas ETLEN}

Frente a otras indagaciones, en el proyecto ETLEN se eligió el mayor número posible de fenómenos diferenciales de tipo fonético y gramatical. Puesto que los diversos trabajos preexistentes eran muy heterogéneos en cantidad y calidad, y no cubrían 
sistemáticamente todo el territorio estudiado, se confeccionó un cuestionario formado por 368 fenómenos diferenciales, dividido en 3 bloques: 111 fenómenos fonéticos y fonológicos; 162 fenómenos de morfosintaxis nominal; y 95 fenómenos de morfosintaxis verbal. Dado que cada fenómeno está ilustrado con varios ítems (ejemplos, palabras o expresiones concretas), el atlas ofrece en total 531 mapas dialectográficos.

Se eligieron 40 puntos o localidades de encuesta. La campaña de encuestación tuvo varias fases y se alargó hasta el año 2012. En cada localidad se contó con entre una y tres personas de más de 65 años, nacidas en el lugar y vecinas de él toda su vida. Cada entrevista duró entre cinco y siete horas, por lo que tuvo que hacerse en varias sesiones. Todas las entrevistas están grabadas. El tratamiento de los datos incluyó tres tipos de operaciones que constituyen las tres secciones del atlas:

(1) Dialectografía. Se muestran los datos lingüísticos en una colección de mapas dialectales, formando un atlas lingüístico de hechura clásica.

(2) Horiometría. Se aplica una metodología estadística que muestra la proporción de rasgos "occidentales" y "orientales" que tiene el habla de cada localidad.

(3) Dialectometría. Se aplica una metodología estadística que mide matemáticamente las semejanzas y diferencias lingüística entre las localidades del territorio estudiado.

Para la sección dialectográfica y horiométrica se usó el programa informático Cartografía Dialectal (CartoDial), creado y registrado por el propio equipo; y para la sección dialectométrica, el programa Visual DialectoMetry (vdm), creado por el equipo del profesor Hans Goebl, de la Universidad de Salzburgo, y adaptado a nuestro proyecto (Goebl, 2011, 2012). 


\section{4.}

\section{Sección dialectográfica}

En ella se sigue la línea de los atlas lingüísticos tradicionales. En total, la sección dialectográfica del atlas ETLEN está formada por 532 mapas en color.

He aquí un ejemplo de mapa dialectográfico: se refiere a un fenómeno fonético-fonológico nunca cartografiado: "mantenimiento o alteración de [wa] en los resultados de la secuencia [ wa]", referido a la pervivencia de la secuencia vocálica -oa-, -uaen el nombre de persona Xuan (del latín Iohanne), o su simplificación en - $a-(X a n)$.

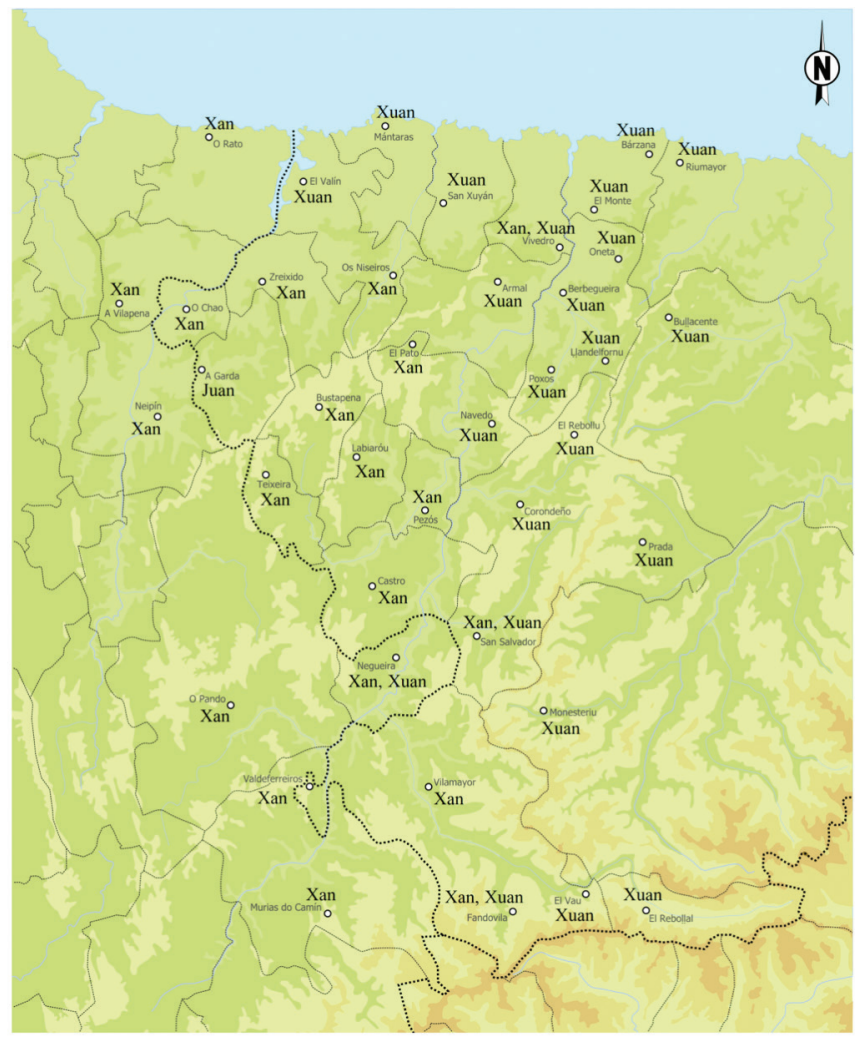

Figura 3. Mapa dialectográfico del fenómeno fonético "mantenimiento o alteración de [wa] en los resultados de la secuencia [ [wa]", ítem "Xuan". 


\section{5.}

\section{Sección horiométrica}

La horiometría es una innovación de este atlas. De hecho, es un neologismo creado por el equipo investigador (griego hórion 'frontera, límite' y métron 'medida'). Definimos la horiometría como la medición de una frontera lingüística basada en la proporción de rasgos adscribibles a cada espacio geolectal (geotipo, dominio) en cada punto del territorio estudiado (Álvarez-Balbuena et al., 2011; Andrés Díaz et al., 2011).

\section{1 .}

\section{Adscripción geotipológica}

La horiometría se basa en el principio de adscripción geotipológica, es decir, en la posibilidad de clasificar muchas geovariantes (rasgos diferenciales) como integrantes de un espacio geolectal. En el atlas etlen estos espacios geolectales son: el occidental; el oriental; y el axial. El espacio occidental se puede identificar, pero no necesariamente, con el dominio gallegoportugués; y lo mismo se puede decir del espacio oriental respecto del asturleonés. El espacio axial viene a coincidir a menudo, pero no siempre, con las hablas de transición que forman el gallego-asturiano. Si un rasgo aparece igual en todos los espacios geográficos, se clasifica como común y no tiene valor diferencial.

A fin de cuantificar la adscripción geotipológica de cada geovariante a un espacio geolectal, nos ayudamos de cuatro parámetros:

$\begin{array}{ll}\text { +Occidental } & \text {-Oriental } \\ \text {-Occidental } & \text { +Oriental } \\ \text {-Occidental } & \text {-Oriental } \\ \text { +Occidental } & \text { +Oriental }\end{array}$

A cada uno de estos parámetros se le asigna un valor. La suma de los cuatro valores no ha de superar 100. Como ejemplo, consideremos el fenómeno "forma del pronombre átono de 3. ${ }^{\text {a }}$ persona del singular en función de complemento directo". Sus geovariantes son o occidental, lo oriental y ol el axial (por ejemplo, véxoo \| 
véxol || véxolo 'lo veo'). En O Rato (Ribadeo) la forma recogida es $o$ (véxoo), de manera que la puntuación es esta:

$\begin{array}{llr}\text { +Occidental } & \text {-Oriental } & 100 \\ \text {-Occidental } & \text { +Oriental } & 0 \\ \text {-Occidental } & \text {-Oriental } & 0 \\ \text { +Occidental } & \text { +Oriental } & 0\end{array}$

En Mántaras (Tapia) la forma registrada es lo (véxolo), luego la puntuación es esta otra:

$\begin{array}{llr}\text { +Occidental } & \text {-Oriental } & 0 \\ \text {-Occidental } & \text { +Oriental } & 100 \\ \text {-Occidental } & \text {-Oriental } & 0 \\ \text { +Occidental } & \text { +Oriental } & 0\end{array}$

En Teixeira (Santalla d'Ozcos) la forma registrada es $l$ (véxol), y por tanto la puntuación es:

$\begin{array}{llr}\text { +Occidental } & \text {-Oriental } & 0 \\ \text {-Occidental } & \text { +Oriental } & 0 \\ \text {-Occidental } & \text {-Oriental } & 100 \\ \text { +Occidental } & \text { +Oriental } & 0\end{array}$

Respecto al fenómeno "conservación o pérdida de la -L- intervocálica del latín”, la geovariante - $\varnothing$ - es occidental y - $l$ - es oriental. Dados los étimos latinos gelare, palu y calĕnte, en A Garda (Taramunde) se constata xear y pau, pero calènte. Por tanto, la puntuación es:

$\begin{array}{llr}\text { +Occidental } & \text {-Oriental } & 66 \\ \text {-Occidental } & \text { +Oriental } & 34 \\ \text {-Occidental } & \text {-Oriental } & 0 \\ \text { +Occidental } & \text { +Oriental } & 0\end{array}$

\section{2.}

\section{Mapas horiométricos}

De las anteriores operaciones se obtienen mapas horiométricos, generados automáticamente; son 35 en total. Un mapa horiométrico muestra la proporción porcentual de rasgos occidentales, 
orientales y (cuando los hay) axiales que caracterizan el habla de cada lugar. Hay dos tipos de mapas horiométricos: los circulares y los poligonales.

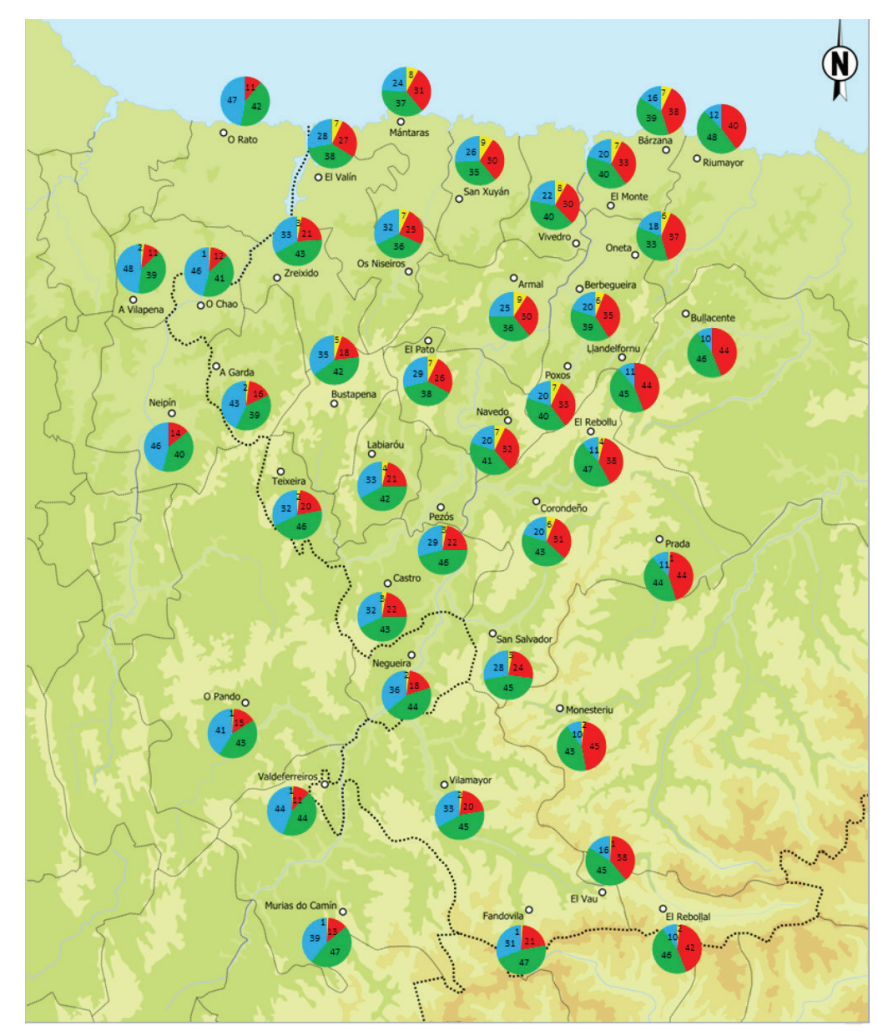

Figura 4. Mapa horiométrico circular referido al total de los 111 fenómenos fonéticofonológicos. Código cromático: azul = occidental, rojo = oriental, amarillo = axial, verde = común.

La figura 4 muestra un mapa horiométrico circular referido a la masa de los 111 fenómenos fonético-fonológicos. Cada diagrama circular o "de tarta" contiene la proporción, en porcentaje, que el habla de cada lugar tiene de "occidentalidad" (azul), "axialidad" (amarillo), "orientalidad” (rojo) y "común" (verde). Por ejemplo, la localidad de Mántaras (Tapia) parece tener, en el análisis de su nivel fonético-fonológico, un 37\% de occidental, un $8 \%$ de axial, un $24 \%$ de oriental y un $37 \%$ de rasgos comunes. 
La horiometría también se puede representar por medio de mapas poligonales, que parcelan el territorio en casillas o polígonos correspondientes a cada uno de los lugares. En el mapa poligonal de la figura 5, referido a la masa de 111 fenómenos fonético-fonológicos, se toman como referencia los geotipos occidental y oriental al mismo tiempo. Los porcentajes aumentan de acuerdo con la «orientalidad». Se aprecia fácilmente la transición gradual de «menor orientalidad» (azul) a «mayor orientalidad» (rojo).

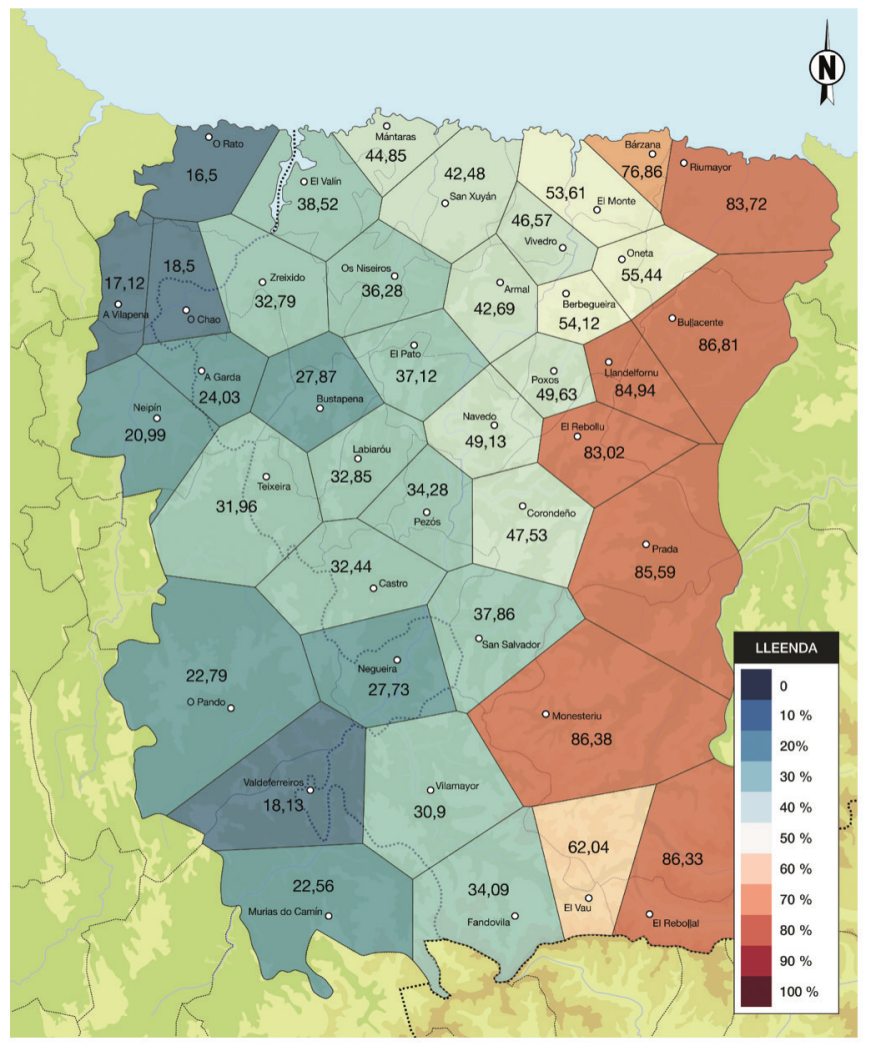

Figura 5. Mapa horiométrico poligonal de referencia occidental / oriental, referido al total de los 111 fenómenos fonético-fonológicos. 


\section{6.}

\section{Sección dialectométrica}

La dialectometría estudia la variación dialectal de un territorio mediante la medición matemático-estadística de las diferencias lingüísticas entre los diversos puntos. La dialectometría no toma como referencia el concepto de frontera ni contempla la adscripción de los rasgos a dominios lingüísticos; lo que busca es simplemente cuantificar las diferencias lingüísticas entre los lugares del territorio. Para ello traduce tales diferencias a unidades de cómputo o recuento (taxats) que permiten cálculos estadísticos. Estos cálculos se trasladan a mapas que representan los valores estadísticos gracias a gamas de colores (Goebl, 2011, 2012). El atlas ETLEN incluye 77 mapas dialectométricos. Hay varios tipos de mapas dialectométricos; nos fijaremos solamente en dos: los sinópticos y los dendrográficos.

\section{1 . \\ Mapas sinópticos}

Los mapas sinópticos son, a su vez, de diversos tipos; uno de ellos, el de punto de referencia, permite contemplar la variación lingüística dentro del territorio desde la perspectiva de una localidad concreta. En la composición de mapas de la figura 6 -referidos al corpus total del etlen- se han elegido distintos pueblos de referencia, recorriendo el contorno del territorio estudiadol.

1 Se ha usado el algoritmo MINMAMAX aplicando una segmentación en 4 intervalos. La trama recta indica la localidad con valores de similitud más altos respecto del punto de referencia; la trama oblicua indica la localidad con valores de similitud más bajos. Cada mapa se acompaña de un histograma de frecuencias y una leyenda, que aquí no reproducimos por falta de espacio. 

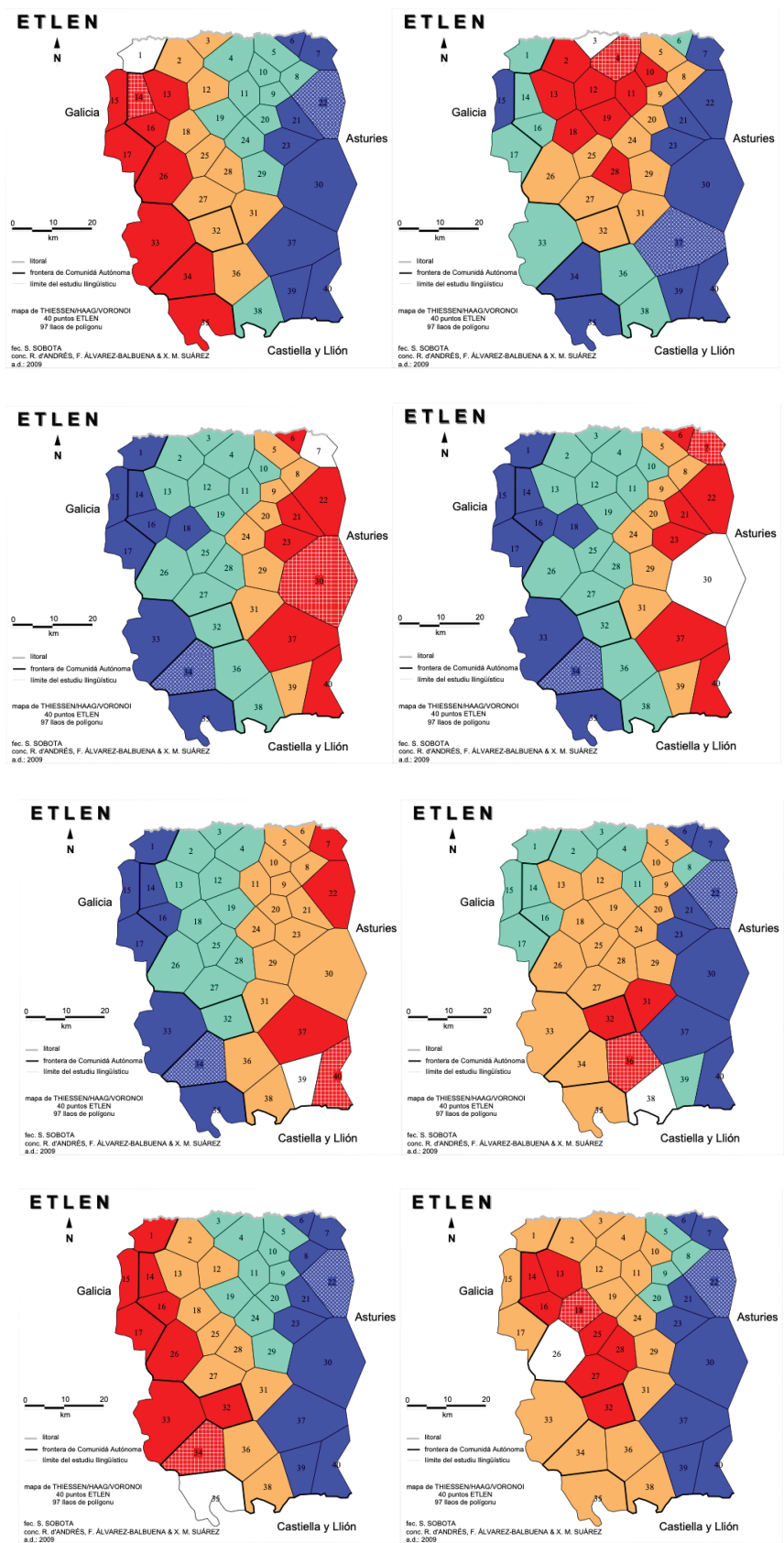

Figura 6. Mapas dialectométricos sinópticos de punto de referencia y cuatro segmentaciones. 
En estos mapas, el rojo indica las localidades más semejantes lingüísticamente al punto de referencia, es decir, las más "amigas"; el color naranja indica localidades también "amigas", pero en menor grado. El color azul oscuro indica las localidades más diferentes lingüísticamente al punto de referencia, es decir, las más "extrañas"; el color azul claro indica localidades también "extrañas", pero en menor grado.

\section{2 .}

\section{Mapas dendrográficos}

El programa vdm ejecuta también análisis aglomerativo o de clústers. Consiste en hacer sucesivas aglomeraciones automáticas de las localidades, basadas en las similitudes estadísticas, de acuerdo con diversos métodos. Estas sucesivas aglomeraciones quedan representadas en un diagrama arbóreo o dendrograma (a la izquierda). Sobre él se puede elegir un corte en dos o más aglomeraciones, llamadas dendremas y que corresponden en el mapa a otros tantos territorios llamados coremas.

El siguiente mapa dendrográfico de la figura $7^{2}$, referido al subcorpus fonético-fonológico, muestra una partición en dos dendremas / coremas, que aparecen con dos colores de valor puramente identificativo (sin valor estadístico). Esta bipartición recuerda mucho la disposición geográfica de los dominios gallegoportugués y asturleonés (téngase en cuenta que este mapa se refiere solo al nivel fonético-fonológico).

Si sobre el mismo mapa hacemos un corte en tres aglomeraciones, en el anterior dendrema rojo aparece una subdivisión (verde) en toda la franja central del territorio estudiado. Esta franja central verde puede interpretarse como la parte del área gallegoportuguesa en la que lo fonético-fonológico es más cercano lingüísticamente al área asturleonesa (figura 8).

2 En este mapa y el siguiente se aplicó el índice relativo de identidad (IRI) y se usó el método de distancia máxima o de Ward. 


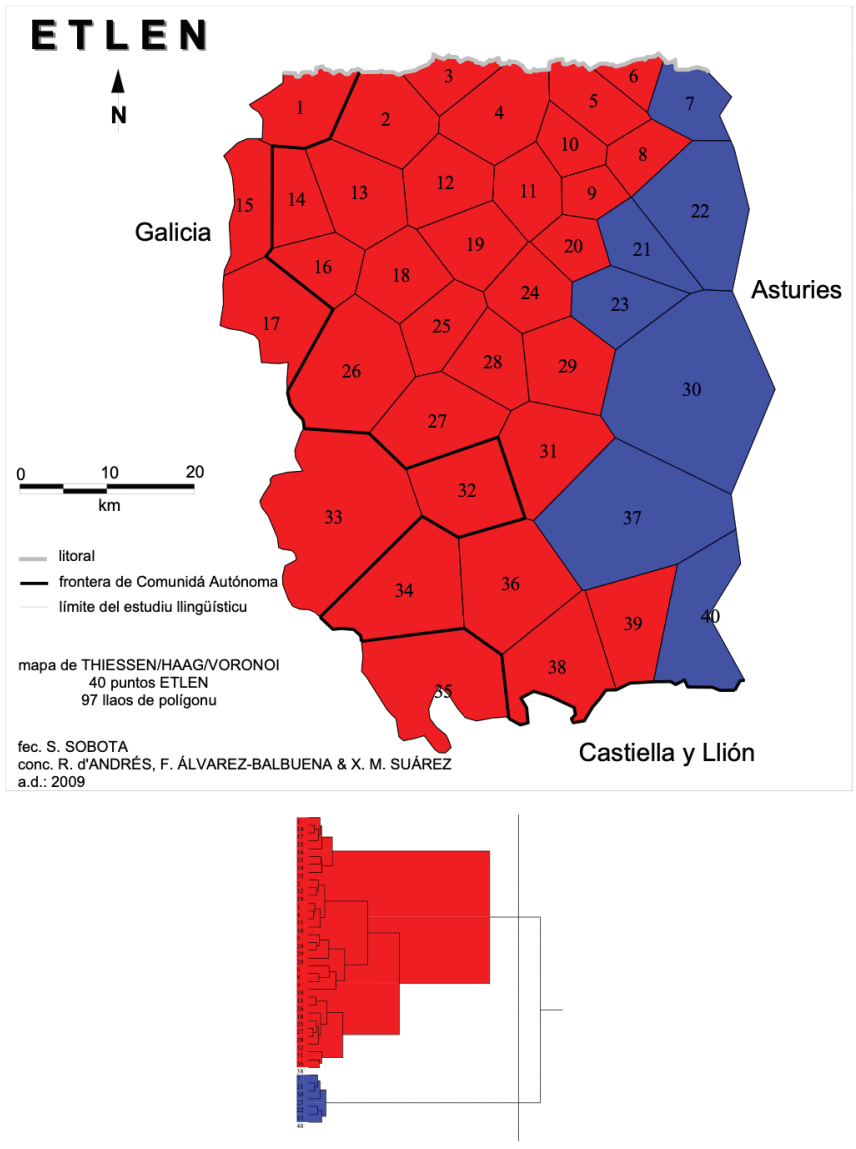

Figura 7. Mapa dialectométrico dendrográfico según el método de Ward y partición binaria, referido al total de los 111 fenómenos fonético-fonológicos. 

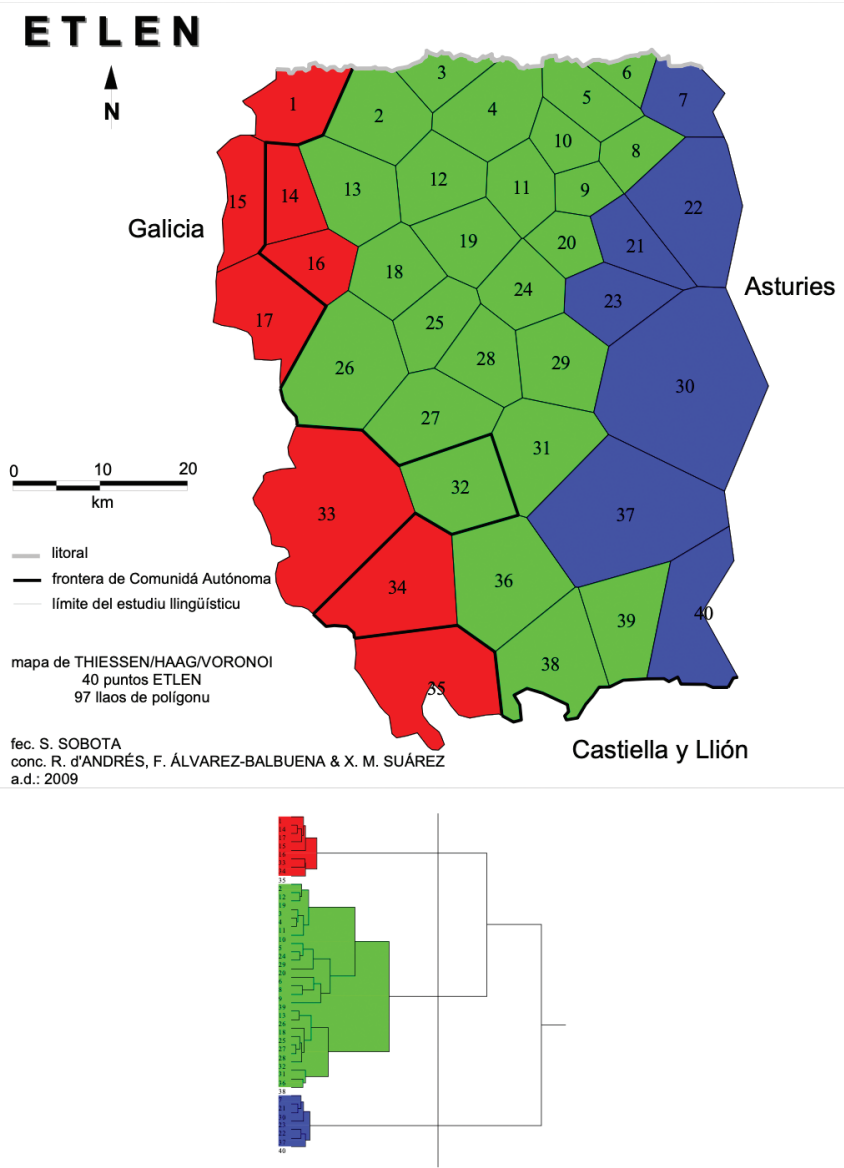

Figura 8. Mapa dialectométrico dendrográfico según el método de Ward y partición ternaria, referido al total de los 111 fenómenos fonético-fonológicos.

\section{7.}

\section{Conclusiones}

- El atlas etlen, con su enorme cantidad de información, puede ser un instrumento que ayude a conocer en más profundidad las características lingüísticas de la zona Eo-Navia como emblemática zona de transición del mundo hispánico y románico. 
- Los métodos aplicados en esta investigación no invalidan el corpus de conocimientos logrados por la dialectología tradicional, sino que vienen a corroborarlo con mayor precisión.

- La metodología desplegada en el etlen sin duda puede ser de gran valor en el estudio de otras conocidas zonas de frontera geolectal, dentro y fuera de la península Ibérica.

\section{Referencias bibliográficas}

Álvarez-Balbuena et. al. (2011) = Álvarez-Balbuena García, F., Andrés Díaz, R. de, Suárez Fernández, X. M., Cueto Fernández, M. (2011), "La «horiometría» o dialectometría de frontera", IX Congreso Internacional de Lingüística General. 21-23 de junio de 2010. Universidad de Valladolid. Actas del congreso, Valladolid: Instituto Castellano y Leonés de la Lengua / Ayuntamiento de Valladolid / Diputación de Valladolid, 107-134.

Andrés, R. de (2011), "Fronteras lingüísticas y geotipos, con atención a la zona Eo-Navia”, en R. de Andrés Díaz (coord.), Lengua, ciencia y fronteras, Uviéu: Ediciones Trabe / Universidá d’Uviéu (Seminariu de Filoloxía Asturiana), 121-152.

Andrés, R. d' (2015), “La frontera ente l'asturianu y el gallegu: elementos pa un análisis horiométricu”, en R. d'Andrés (coord.), Manuel Menéndez y l'Asturies occidental, Uviéu: Universidá d'Uviéu / Ediciones Trabe, 45-76.

Andrés Díaz et al. (2013) = Andrés Díaz, R. de, Álvarez-Balbuena García, F., Cueto Fernández, M., Suárez Fernández, X. M. (2013), "About the concept of «geodifferential feature» between linguistic varieties in contact", en E. Carrilho, C. Magro y X. Álvarez (eds.), Current Approaches to Limits and Areas in Dialectology, Newcastle upon Tyne: Cambridge Scholars Publishing, 85-122.

Andrés Díaz et al. (2017) = Andrés Díaz, R. d' (dir.), Álvarez-Balbuena García, F., Suárez Fernández, X. M., Rodríguez Monteavaro, M. (2017), Estudiu de la transición llingüistica na zona Eo-Navia, Asturies (ETLEN). Atles llingüísticu dialectográficu - horiométricu - dialectométricu, Uviéu: Trabe / Universidá d'Uviéu. 
Goebl, H. (2011), "Áreas, fronteras, similitudes y distancias: lección breve de geolingüística cuantitativa”, en R. de Andrés (coord.), Lengua, ciencia y fronteras, Uviéu: Ediciones Trabe / Universidá d'Uviéu, 11-34.

Goebl, H. (2012), "Introduction aux problèmes et méthodes de l'«École dialectométrique de Salzbourg» (avec des exemples gallo-, italo- et ibéromans)", en X. A. Álvarez Pérez, E. Carrilho y C. Magro (eds.), Proceedings of the International Symposium on Limits and Areas in Dialectology (LimiAr), Lisbon, 2011, Lisboa: Centro de Linguística da Universidade de Lisboa, 117-166.

Menéndez Pidal, R. (1906), "El dialecto leonés", Revista de Archivos, Bibliotecas y Museos, 3a época, año x, 2-3, pp. 128-172.

Munthe, Å. W. (1887), Anteckningar om Folkmålet i en trakt af Vestra Asturien, Uppsala: Almqvist \& Wiksell. [Traducción (1988): Anotaciones sobre el habla popular de una zona del Occidente de Asturias, versión castellana de B. Pallares, edición de A. M. Cano, Uviéu: Universidá d'Uviéu]. 